ing is that nanoscale indium particles seem to self-organize into specific sizesmagic number sizes," Allen said. In the October 23 issue of Physical Review Letters, Efremov, Schiettekatte, and co-workers show, using nanocalorimetry, that the particles appear to self-assemble into special "magic" sizes, where each size differs incrementally by a single complete shell of atoms.

STEFFEN K. KALDOR

\section{Diffraction-Free Propagation of Rayleigh Waves Observed along Solid Spherical Surfaces}

Vibration and surface acoustic wave propagation in elastic spheres has been studied extensively, especially in seismology, where an elastic sphere is viewed as the simplest model of the Earth. Recent experiments have shown that the method by which surface waves propagate along a sphere depends on whether they arise from a point source or a line source. In seismology studies, where the source is orders of magnitude smaller than the Earth's radius, a point source assumption is valid, and the wave behavior is well understood. However, researchers Yusuke Tsukahara and Noritaka Nakaso of Toppan Printing Company Ltd. and Hideo Cho and Kazushi Yamanaka of the Department of Materials Processing at Tohoku University observed earlier this year that as the radius of the sphere becomes comparable in size to the source, markedly different wave-propagation behavior occurs that cannot be explained by a point-source analysis, suggesting diffraction-free propagation [Appl. Phys. Lett., 76 (2000) p. 2797]. Now, in the October 30 issue of Applied Physics Letters, the researchers present a model, along with supporting experimental evidence, to explain this observed phenomenon.

When a point force is applied to the surface of an elastic sphere at the south pole, Rayleigh waves, in addition to bulk waves, propagate along the surface in all directions. As the wave front crosses the equator, it converges toward the north pole and then diverges back toward the south pole; this process repeats indefinitely. Experiments carried out with a 20-mm-diameter sphere heated by a laser beam focused to a small spot demonstrate this behavior. However, experiments employing an 8$\mathrm{mm}$-diameter sphere heated by a line source consisting of a traveling optical inteference pattern show surface waves which travel within a narrow path over the spherical surface rather than spreading out, that is, diffraction-free propagation.

To explain this phenomenon, Tsukahara and co-workers postulate that a line source of finite length, located along the equator, generates a beam of Rayleigh waves that undergo two effects that balance, if the line source length is appropriately chosen. The first effect, due to the curvature of the spherical surface, causes the Rayleigh waves to converge toward the poles, while the second effect, diffraction from a finite aperture, causes the Rayleigh waves to spread out over the surface of the sphere. When these two effects cancel, diffractionfree propagation of a finite beam width is realized.

To verify their theory, the researchers fabricated and polished an 80-mm-diameter glass ball. An ultrasonic transducer

ADVANGED MIGROANALYYSIS TEGHNOLOGY AJ AN AFFORDABLE PRIGE

MIJAOAMALYZEA OFFEABS
$\triangle$ Fast Report Generation
A Same Algorithms as Phoenix
A Fully Upgradable to Phoenix
$\Delta$ Interface to Existing or New Detectors

EDAX FEATUAES:

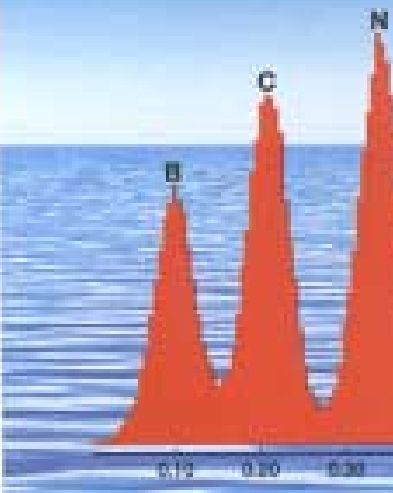

$\checkmark \mathrm{PCl}$ Bus (32 bit)

Architecture

$\checkmark$ Windows 98 or Windows NT

$\checkmark$ Digital Processing Standard

7 Superior Service \& Support
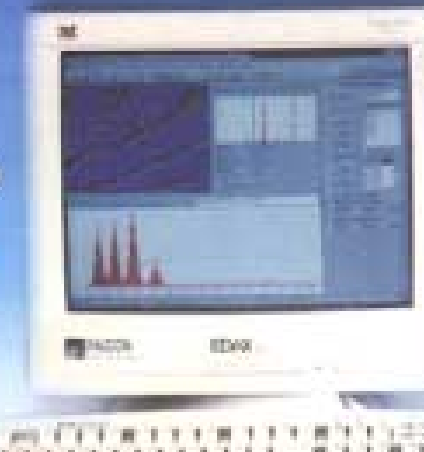

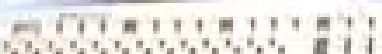

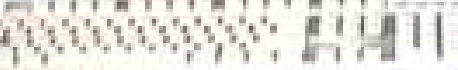

Falcon system

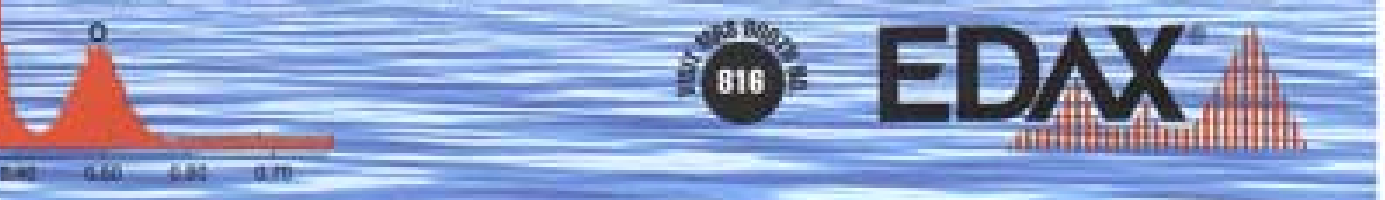

Circle No. 6 on Inside Back Cover 
of 1-3 composite made of epoxy and PZT was cut into a strip $(1.5 \mathrm{~mm}$ long $\times 20$ $\mathrm{mm}$ wide $\times 1.4 \mathrm{~mm}$ thick) and glued onto the glass ball. Using a pulse generator connected to the transducer to provide an electrical pulse, a Rayleigh wave with frequency $\sim 1.1 \mathrm{MHz}$ was excited. The transducer was subsequently used to monitor the resulting wave propagation. Waveform measurements show that the width of the Rayleigh wave was $\sim 20 \mathrm{~mm}$ (the width of source transducer) and support the model of diffraction-free propagation of the Rayleigh wave around a solid sphere. The single transducer generated both surface and bulk acoustic waves. Other measurements were performed in which a second transducer was placed collinear with the first and excited with opposite polarity. In these experiments, the ratio of surface-to-bulk acoustic waves was strongly enhanced.

Work is under way to calculate the beam propagation of Rayleigh waves for further comparison with experiment. According to Tsukahara, this phenomenon is probably not limited to Rayleigh waves. "We anticipate that the same mechanism of diffraction-free propagation will be applicable to other waves, such as light and quantum mechanical wave functions, given that the waves are confined to the surface of a sphere with an appropriate diameter-to-wavelength ratio."

STEFFEN K. KALDOR

\section{Abnormal Oxidation of $\mathrm{TiSi}_{2}$ in Gate Stacks Found at $750-850^{\circ} \mathrm{C}$}

A team of researchers at the Hyundai Electronics Industries Company Ltd. has recently reported abnormal oxidation behavior of $\mathrm{TiSi}_{2}$ sidewalls in patterned $\mathrm{TiSi}_{2}$ /polysilicon gate stacks at temperatures above $800^{\circ} \mathrm{C}$. The enhanced oxidation of the $\mathrm{TiSi}_{2}$ film was attributed to structural aspects, as it was not observed in unpatterned gate stacks. This suggests that the gate reoxidation of $\mathrm{TiSi}_{2} /$ polysilicon stacks has to be performed at temperatures below $750^{\circ} \mathrm{C}$.

"Low-resistivity gate electrodes are often required for device performance, as silicon devices are scaled down. A common approach is to adopt a structure that is composed of a low-resistivity silicide and doped polysilicon (polycide structure)," said Dae-Gyu Park in the November issue of Electrochemical and Solid-State Letters. Gate reoxidation after the patterning process is necessary to increase the reliability of devices. While recent experiments on $\mathrm{WSi}_{2} /$ polysilicon gate stacks showed oxidation of the metal instead of the silicon at low temperatures, In-Seok Yeo and his co-workers found that
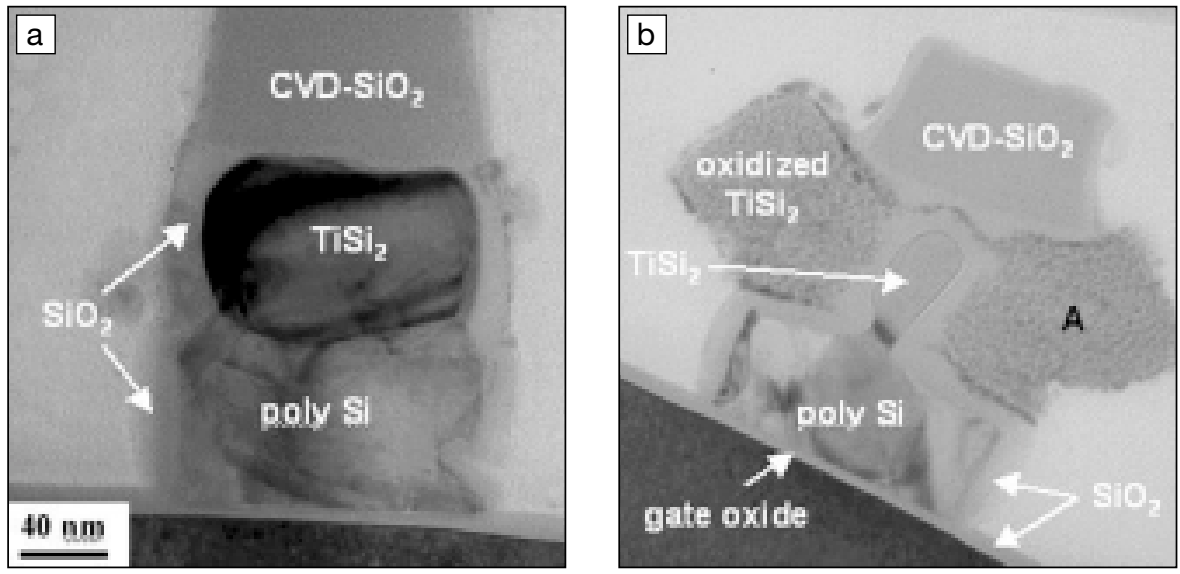

Figure 1. Transmission electron microscopy images of samples oxidized at (a) $750^{\circ} \mathrm{C}$ and (b) $850^{\circ} \mathrm{C}$.

$\mathrm{TiSi}_{2} /$ polysilicon gate stacks displayed abnormal oxidation behavior at high temperatures.

A $900-\AA$-thick film of in situ phosphorus-doped polysilicon was deposited on a 50 - $\AA$-thick gate oxide, followed by deposition of an $800-\AA$-thick film of $\mathrm{TiSi}_{2}$. A 20-s anneal at $850^{\circ} \mathrm{C}$ was performed in order to obtain the desired low-resistivity C54- $\mathrm{TiSi}_{2}$ phase as confirmed by x-ray diffraction. The stack was patterned after deposition of a hard oxide mask layer by etching with $\mathrm{Cl}_{2}$ and $\mathrm{O}_{2}$ and reoxidized in a dry atmosphere at temperatures between $730^{\circ} \mathrm{C}$ and $850^{\circ} \mathrm{C}$. Films treated at $730-750^{\circ} \mathrm{C}$ showed no gate line deformation, whereas the samples heated to above $800^{\circ} \mathrm{C}$ exhibited strong swelling of the sidewalls.

"We were surprised when we observed the severe deformation of the gate sidewalls after oxidation at 800 or $850^{\circ} \mathrm{C}$, said Se-Aug Jang. "From the reported oxidation behavior of other silicide/polysilicon structures, we expected to see abnormal oxides at low temperatures as well. However, the results were perfectly opposite. It was even more interesting that the unpatterned $\mathrm{TiSi}_{2}$ / polysilicon stacks did not show the abnormal oxidation even at high temperatures at all."

The group carried out transmission electron microscopy (TEM) studies to gain a detailed understanding of the oxidation process. While the films annealed at low temperatures showed two adjacent layers of polysilicon and $\mathrm{TiSi}_{2}$ surrounded by a layer of $\mathrm{SiO}_{2}$ (see Figure 1a), four different regions were observed for the hightemperature samples. The $\mathrm{TiSi}_{2}$ film appeared to have shrunk considerably and was surrounded and separated from the polysilicon by $\mathrm{SiO}_{2}$. Furthermore, regions of a composite were found to extrude from the original sidewall positions (see Figure 1b). This composite, marked " $A$ " in Figure $1 b$, consisted of nanocrystalline $\mathrm{TiO}_{2}$ in an amorphous $\mathrm{SiO}_{2}$ matrix, which was identified from a high-resolution TEM study.

"We believe that this phenomenon is related to the simultaneous exposure of $\mathrm{TiSi}_{2}$ and polysilicon to the oxidant, as we did not observe it in unpatterned films," said Yeo. "We have ruled out any effects from polysilicon film thickness as well as etching damage, and we will conduct further studies that will hopefully help to clarify the mechanism."

CORA LIND

\section{$\mathrm{Si} / \mathrm{TiN}$ Nanocomposites Exhibit Stable Capacities as Anode Material for Li-Ion Batteries}

Research efforts have been directed toward the development of alternative anode materials for Li-ion batteries to achieve higher capacities than possible with traditional graphite anodes. Many projects have focused on intermetallic compounds containing lithium because of their high gravimetric/volumetric capacities. However, lithium alloys present problems, due to a large volume change during charge/discharge that results in a cracking of the anodes and loss of capacity during cycling. To avoid this problem, composite microstructures of a finely dispersed interconnected active phase in an inactive host structure have been explored. In the November issue of Electrochemical and Solid-State Letters, researchers from Carnegie Mellon University (CMU) and Blomgren Consulting Services Ltd. have reported that their composites, which consist of fine Si particles in a TiN matrix, exhibited stable capacities of $\sim 300 \mathrm{mAh} / \mathrm{g}$ and good capacity retention. 